\title{
Telemedicine Application in Toxicology
}

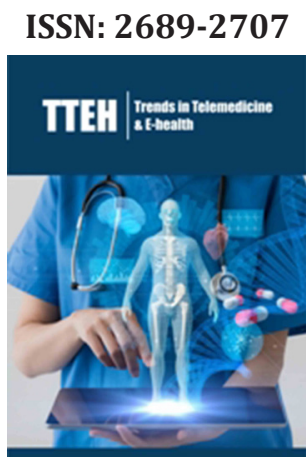

*Corresponding author: Sadrieh Hajesmaeel Gohari,

${ }^{1} \mathrm{PhD}$ candidate of Health Information Management ,Department of Health Information Management, School of Allied Medical Sciences, Tehran University of Medical Sciences

(TUMS),Tehran, Iran

${ }^{2} \mathrm{PhD}$ candidate of Health Information Management, Medical Informatics Research Center, Institute for Futures Studies in Health, Kerman University of Medical Sciences, Kerman, Iran

Submission: 望 June 19, 2019

Published: 侮June 24, 2019

Volume 1 - Issue 4

How to cite this article: Sadrieh Hajesmaeel Gohari. Telemedicine Application in Toxicology. Trends Telemed E-Health. 1(4). TTEH. 000517. 2019. DOI: 10.31031/TTEH.2019.01.000517

Copyright@ Sadrieh Hajesmaeel Gohari, This article is distributed under the terms of the Creative Commons Attribution 4.0 International License, which permits unrestricted use and redistribution provided that the original author and source are credited.

\section{Sadrieh Hajesmaeel Gohari ${ }^{1,2 *}$}

${ }^{1} \mathrm{PhD}$ candidate of Health Information Management ,Department of Health Information Management, School of Allied Medical Sciences, Tehran University of Medical Sciences (TUMS), Tehran, Iran

${ }^{2} \mathrm{PhD}$ candidate of Health Information Management, Medical Informatics Research Center, Institute for Futures Studies in Health, Kerman University of Medical Sciences, Kerman, Iran

\section{Opinion}

Poisoning is an abnormality in the body that is caused by the entry of foreign substances such as toxins and drugs into the body. The entry of poison into the body occurs in several ways, such as breathing gases and toxic vapors, eating and drinking toxic substances, skin contact, and blood transfusions [1]. Poisoning is of different kinds based on the type of toxic substance, which includes food, heavy metals, pesticides, radioactive substances, and drugs. Poisoning is the third cause of death and unintentional injuries in the world [2]. According to the WHO statistics in 2012, approximately 193200 people died from unintentional poisoning and one million people from suicide with toxic substances yearly [3].

Poisoning is an acute disease requiring immediate intervention, but lack of specialized hospital and medical toxicologists in cities could prevent timely intervention. When a physician working in a deprived area faces such a disease, if s/he does not know and cannot take the appropriate treatment, s/he should send the patient to the specialized center, which might lead to the loss of time for treatment. In such a situation, if a doctor can consult with a medical toxicologist, many patients may be treated and continue to live with minimum complications.

In recent years, progress in software, hardware, and wireless infrastructure as well as availability of computers, mobile phones, and tablets has led to the use of telemedicine services in various areas. Telemedicine refers to the use of information and communication technology (ICT) to provide medical and therapeutic services for distant and impoverished regions. This technology is done in three ways, such as store and forward, real time, and virtual, and has different subdomains. Teletoxicology is one of the subdomains of telemedicine that provides toxicology services for the distant area. Teletoxicology may be useful due to high prevalence of poisoning and lack of medical toxicologist in specialized centers in the provinces. For many poisoned patients with low risk poisoning, a brief examination by a medical toxicologist using teletoxicology services could significantly reduce the number of referrals to specialized centers, length of hospitalization, and relevant costs.

Teletoxicology services are done by various technologies from simple to complex. In Saudi Arabia, a teletoxicology system was used via a telephone. In this system, patients or their families could contact the number 937, a poison control center, and consult with medical toxicologists on the management of the poisoned patient [4]. In the United States, electronic mail has been used as teletoxicological method to provide teleconsultation with a medical toxicologist for the military personnel in Afghanistan and Iraq. In this system, when physicians working in war zones need to consult with medical toxicologists, they send the encrypted email containing the patient's information to the medical toxicologists and the toxicologists send back the answer after the maximum of 24 hours via email [5]. In another project in the United States, Google Glasses were used to provide teleconsultation of poisoning. In this project, the counseling physician examined the patient by wearing glasses equipped with a webcam camera, and, then, all the images from the examinations were sent to the medical toxicologist simultaneously [6]. 
Nevertheless, use of simple technologies such as telephones for consultation on poisoning is more common. However, it is necessary for toxicologists to observe physical examination and electrocardiogram image of the poisoned patient in order to provide proper consultation. Therefore, using real-time audio-visual telemedicine technologies improves teletoxicological consultation Audio-visual technologies have the ability to support the patient's examination virtually and provide personalized management decisions compared to the traditional telephone consultation. The results of the study showed that the use of head-mounted devices (HMD), such as Google Glasses, as real-time video telemedicine could be an effective tool for providing teletoxicological services [6].

Despite great advancement in ICTs and the aids of these technologies in the provision of telemedicine services, especially in the field of poisoning, the use of these technologies has been reported very low in poisoning. Probably, one of the main obstacles to the widespread use of teletoxicology, similar to other telemedicine services, is the reimbursement issue. Although efforts have been made to address the reimbursement of teletoxicological services [7], further and deeper research on the successful design and implementation of teletoxicology is necessary.

\section{References}

1. https://www.who.int/environmental_health_emergencies/poisoning/ en/

2. Vassilev ZP, Marcus SM (2007) The impact of a poison control center on the length of hospital stay for patients with poisoning. Journal of Toxicology and Environmental Health, Part A 70(2): 107-110.

3. WHO. Poisoning prevention and management.

4. Al Mazroua M, Issa SY, Hafez EM (2016) Tele-health: Bridging the gap between the need for rapid toxicology consultation and shortage in poison control centers-a unique experience in Dammam poison control center. International Journal of Pharmacology and Toxicology 4(1): 5965.

5. Maddry JK, Sessions D, Heard K, Lappan C, McManus J, et al. (2014) Wartime toxicology: Evaluation of a military medical toxicology telemedicine consults service to assist physicians serving overseas and in combat (2005-2012). Journal of medical toxicology 10(3): 261-265.

6. Chai PR, Babu KM, Boyer EW (2015) The feasibility and acceptability of Google Glass for teletoxicology consults. Journal of Medical Toxicology 11(3): 283-287.

7. Crane PW, Wiegand TJ, Kamali M, Reif M, Wratni R, et al. (2018) Telemedicine delivery and successful reimbursement in toxicology. Journal of Medical Toxicology 14(3): 242-247.

For possible submissions Click below: 